\title{
Multiple aneurysms due to intimomedial mucoid degeneration: a short presentation
}

\begin{abstract}
H Gajjar, MB ChB, FCRad (D) SA
S J Beningfield, $M B C h B$, FFRad (D) SA

Division of Diagnostic Radiology, Groote Schuur Hospital and University of Cape Town

N G Naidoo, MB ChB, FCS (SA)

Vascular Unit,Division of General Surgery, Groote Schuur Hospital, Cape Town

Ahmed Motala, MB ChB

Division of Anatomical Pathology, Groote Schuur Hospital and National Health Laboratory Service, Cape Town

\section{Abstract}

A 17-year-old girl presented with abdominal pain due to contained rupture of a left common iliac artery aneurysm. This was accompanied by abdominal aortic and superior mesenteric artery aneurysms. Despite emergency vascular surgery, the patient died a week later. Post-mortem examination revealed intimomedial mucoid degeneration. This rare condition has been described in predominantly South African black patients, especially females, and occurs at a younger age than degenerative aortic aneurysms.
\end{abstract}

\section{Introduction}

Despite a number of case reports and series, general scepticism persists regarding the acceptance of intimomedial mucoid degeneration (IMMD) as a distinct clinico-pathological entity. This report aims to increase the awareness of IMMD and revisit the distinctive clinical, radiological and pathological features peculiar to this condition. IMMD is a rare degenerative vasculopathy of uncertain aetiology, described predominantly in South African black females. It affects the abdominal aorta most frequently but may also have extra-aortic involvement.

\section{Case report}

A 17-year-old black girl presented to a peripheral hospital with a oneweek history of severe abdominal pain, weight loss, swelling of her left leg, and weakness of both lower limbs, worse on the left. She had no significant prior medical or surgical history of note, except for an appendicectomy. A family history of vascular disease was not elicited. On clinical examination, a tender pulsatile abdominal mass was palpable, with marked oedema of the left lower limb. Reduced femoral pulses with clinical features of acute limb ischaemia were present involving both lower limbs, consistent with Rutherford grade 2B (parasthesia and paresis).

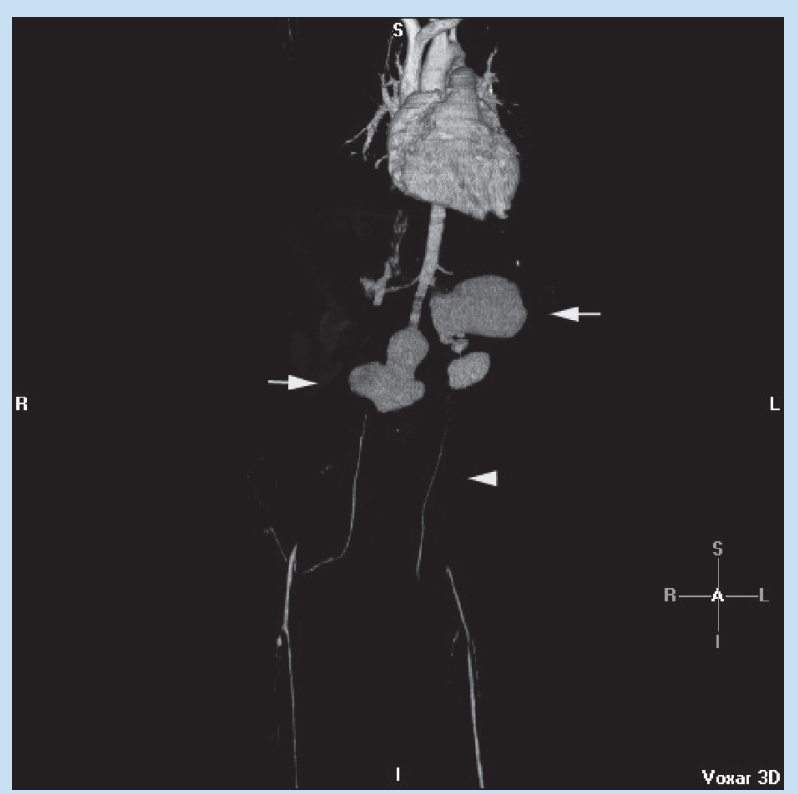

Fig. 1. CT angiogram of thorax and abdomen, surface shaded display. The abdominal aortic aneurysm is seen in the midline and the superior mesenteric artery aneurysm on the left (arrows). The iliac arteries are occluded with reconstitution of the common femoral arteries via collateral vessels (arrowhead).

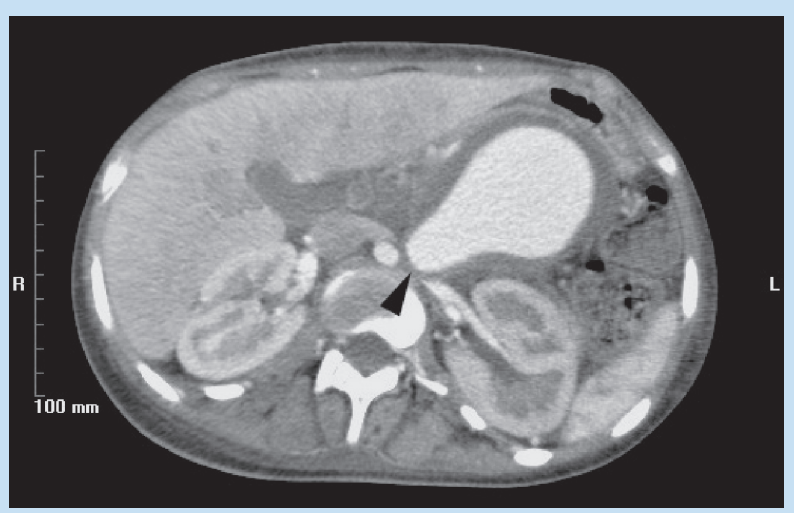

Fig. 2. CT angiogram indicating an aneurysmal superior mesenteric artery (arrowhead). The proximal segment of the abdominal aorta is of normal calibre.

Laboratory investigations indicated a raised white cell count of $22.64 \times 109 / 1$ and reduced haemoglobin of $7.8 \mathrm{~g} / \mathrm{dl}$. The erythrocyte sedimentation rate (ESR) was normal. Other haematological parameters indicated that a disseminated intravascular coagulopathy (DIC) was present. Of note was a normal autoimmune screen with the exception of a positive result for lupus anticoagulant; this was difficult to interpret as 


\section{PICTORIAL ESSAY}

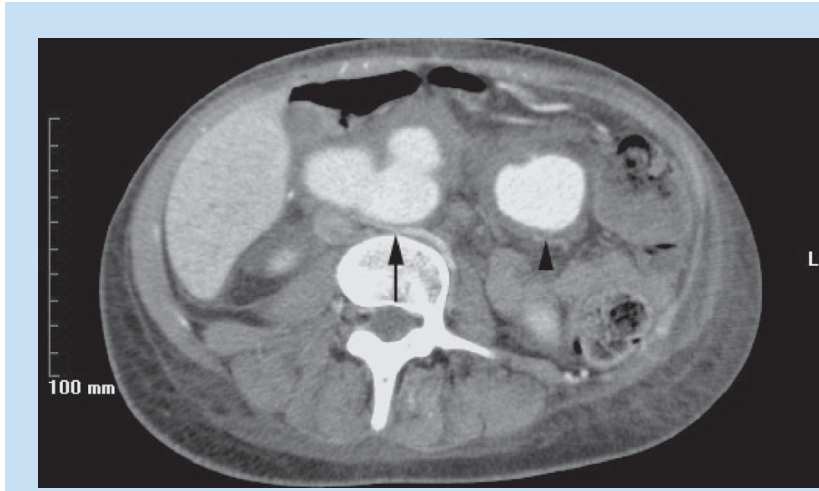

Fig. 3. CT angiogram inferior to Fig. 2 indicating aneurysmal superio mesenteric artery (arrowhead) and abdominal aorta (arrow).

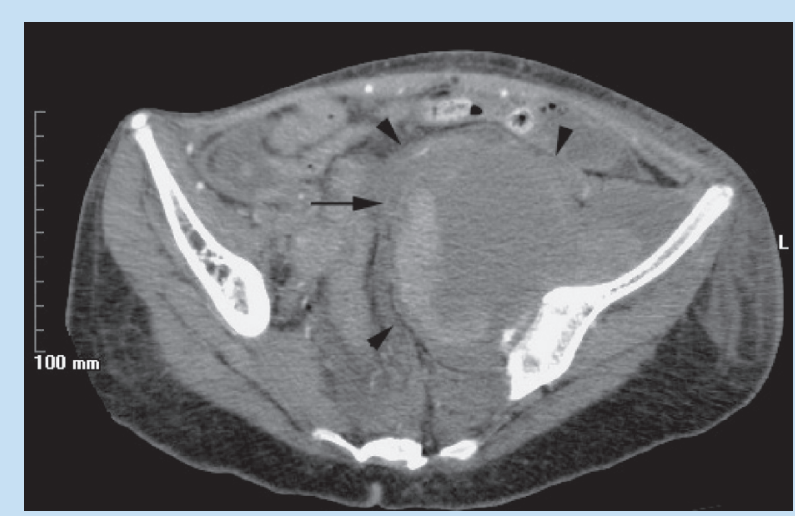

Fig. 4. CT angiogram. A large pelvic haematoma is present (arrowheads) due to contained rupture of the left common iliac artery aneurysm. The right common iliac artery is occluded. Active arterial haemorrhage is visible (arrow).

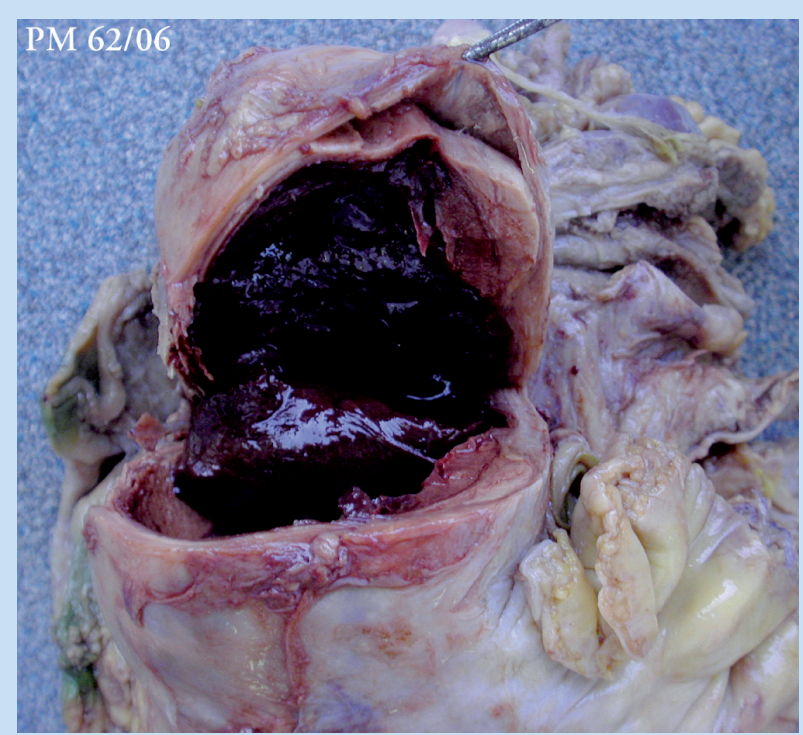

Fig. 5. Superior mesenteric artery aneurysm with post-mortem 'redcurrant jelly' blood clot. Minimal antemortem thrombus present.
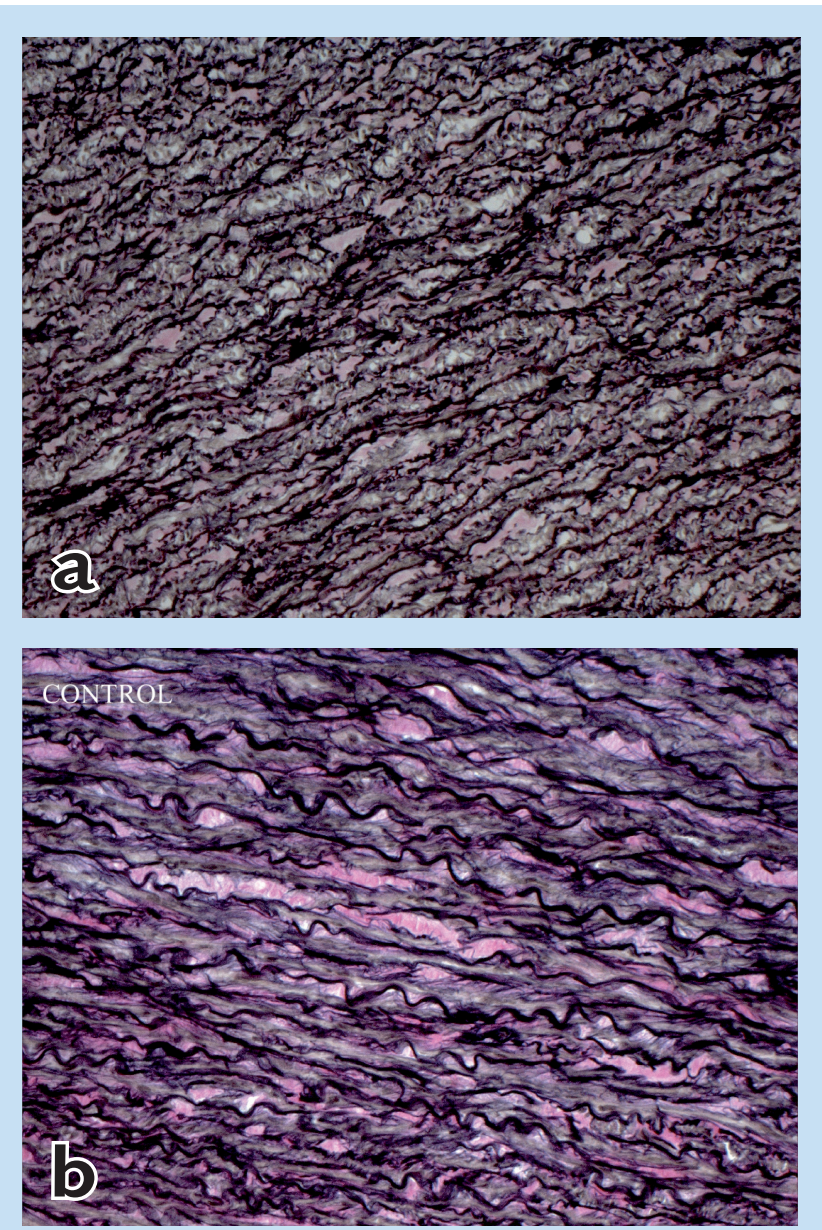

Figs. $6 a$ (patient) and $6 b$ (control) (microscopy). Microscopic specimen of aortic wall using an Elastic Von Gieson (EVG) stain, showing irregular fragmented intimal and medial elastin fibres, relative to an age-matched control.

false positives occur in DIC. Also of note was a normal fibrinogen level which can be seen early in the course of a DIC.

Ultrasound examination revealed an $8 \mathrm{~cm}$-diameter abdominal aortic aneurysm (AAA) and confirmed the presence of a left ileo-femoral deep vein thrombosis. A computerised tomographic angiogram (CTA) of the chest and abdomen revealed an infra-renal aortic aneurysm measuring $5 \mathrm{x} 8 \mathrm{~cm}$ in diameter, as well as a superior mesenteric aneurysm measuring $7 \times 8 \mathrm{~cm}$ in diameter (Figs 1 - 3). The left common iliac artery was also aneurysmal with a contained rupture resulting in a large pelvic retroperitoneal haematoma of $9 \times 13 \mathrm{~cm}$ in diameter (Fig. 4). The right common iliac artery and both external iliac arteries were occluded and reconstituted at the common femoral arteries via collaterals bilaterally. The other major abdominal arteries were normal. Multiple paratracheal lymph nodes were also identified.

The patient underwent complex emergency vascular surgery involving aorto-bifemoral bypass grafting, exclusion bypass grafting of the superior mesenteric aneurysm, reimplantation of the inferior mesenteric artery, as well as bilateral four-compartment calf fasciotomies. The patient was discharged from the intensive care unit 5 days post surgery but arrested in the ward 2 days later. 


\section{PICTORIAL ESSAY}
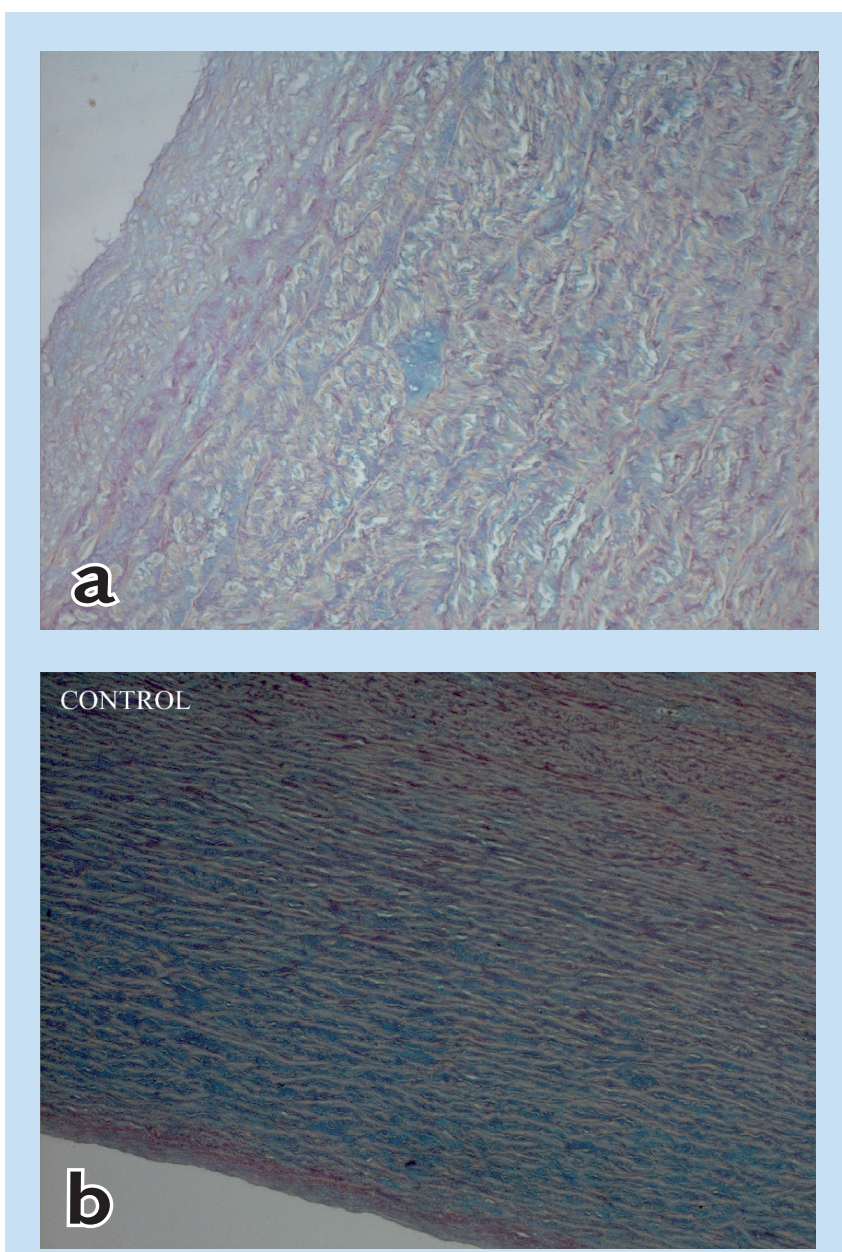

Figs. 7a (patient) and 7b (control) (microscopy). Microscopic specimen of aortic wall using an Alcian Blue stain to demonstrate intimal and medial accumulations of mucoid material, relative to an age-matched control.

\section{Post-mortem findings}

\section{Gross examination}

Post-mortem examination revealed multiple arterial aneurysms with involvement of, inter alia, the abdominal aorta, and superior mesenteric and common iliac arteries (Fig. 5). The cerebral and coronary circulation was normal except for ectasia of the right internal carotid artery. The aneurysms were fusiform in appearance and contained minimal antemortem thrombus. Other findings included mediastinal tuberculous lymphadenitis, left chronic pyelonephritis and mild atherosclerosis.

\section{Histopathological findings}

Specimens of the aneurysms and non-aneurysmal ectatic vessels revealed abnormal accumulations of Alcian Blue positive acidic mucopolysaccharide within the intima and media. In addition, fragmentation and loss of medial elastin fibres was evident within elastic arteries. Significantly, these findings were also seen in vessels unaffected by secondary fibroinflammatory changes in the peri-aortic adventitia, and were interpreted as a response to rupture and false aneurysm formation. The features were consistent with IMMD (Figs 6 and 7).

\section{Discussion}

IMMD is a rare vascular condition that affects predominantly the abdominal aorta, but can also involve other large arteries including the superior mesenteric artery, carotid, subclavian and iliac arteries. ${ }^{1}$ Extra-aortic involvement may also occur in the absence of aortic disease. ${ }^{2}$ The aneurysmal morphological characteristics may be fusiform, saccular or both. IMMD was first described in South Africa ${ }^{1}$ and has been reported exclusively in black patients, the majority being young females. Thirty-one confirmed cases are recorded in the South African literature. ${ }^{1,3}$ However, this vascular pathology is not unique to the South African black population. Isolated cases have been reported outside South Africa, with single cases reported in Uganda ${ }^{4}$ and India. ${ }^{5}$ The population affected is younger than that for degenerative AAA, with an average age of 51.5 years. ${ }^{1}$ Although the exact cause of IMMD is uncertain, it may occur secondary to elastic tissue degeneration or as a result of a primary abnormality of ground substance. ${ }^{2}$ An intriguing possibility raised by this case is the possible existence of trigger factors which resulted in the characteristic clinical and pathological manifestations. These may include a familial predisposition or associated chronic illness e.g. tuberculosis.

Patients with aortic IMMD usually present as a result of local symptoms related to the aneurysms, such as backache and abdominal pain. ${ }^{6}$ Acute lower limb ischaemia due to dissection has been described. ${ }^{1} \mathrm{~A}$ number of cases have been complicated by primary fibrinolysis, which has been reported following surgical manipulation of aneurysms and postulated to be due to release of fibrinolytic enzymes. ${ }^{1}$ This complication did not manifest in our case, with the DIC presumably due to haemorrhage from the large contained rupture of the left common iliac artery aneurysm.

Complications as seen in other degenerative aneurysms can occur, such as rupture or a contained leak. Surgical management does not differ from that of other aneurysmal conditions, although the vessels may be more friable, resulting in technically challenging surgery. Prognosis is principally affected by the nature of the patient's presentation and extent of aneurysmal disease. Acute presentation with aneurysmal complications results in higher morbidity and mortality.

Duplex doppler ultrasound and multidetector CT angiography are the chief imaging modalities used. Digital subtraction angiography can be utilised, but is less commonly required for diagnostic purposes. Interrogation of the subclavian, common carotid, common iliac and superior mesenteric arteries for aneurysmal involvement is essential. Previous reports have suggested that a feature suggesting this condition is lack of thrombus within the aneurysm. In our case, a thin rim of thrombus was present in both the aortic and superior mesenteric aneurysms.

Clinical and imaging differential diagnoses include Takayasu's arteritis, Marfan's syndrome, Ehlers-Danlos syndrome, HIV arteriopathy, TB aortitis and idiopathic aortitis. However, occlusive disease is uncommon in Marfan's and Ehlers-Danlos syndrome, as are signs of systemic inflammation.

The histological findings include intimal thickening, with accumulation of pools of mucopolysaccharide material within the intima and media with an associated loss of elastin fibers. ${ }^{7}$ The presence of similar changes in non-aneurysmal vessels is consistent with the hypothesis that 


\section{PICTORIAL ESSAY}

these changes precede aneurysm formation. ${ }^{1}$ The histological differential diagnosis includes Erdheim's cystic medial necrosis, which involves the media exclusively and may occur in isolation or as a component of Marfan's syndrome.

In conclusion: IMMD should be suspected when aortic or large vessel aneurysmal disease is detected in young black patients. Lessthan-expected or absent thrombus within the aneurysm would provide a further clue on imaging. At surgery, meticulous technique is required as the vessel wall is often thin and friable. The coagulation profile must be meticulously assessed and aggressively corrected, given the reports of primary fibrinolysis induced at surgery. Further research is necessary to define risk factors and to elucidate possible triggers for this potentially fatal degenerative vasculopathy.
1. Abdool-Carrim A, Robbs JV, et al. Aneurysms due to intimomedial mucoid degeneration. Eur J Vasc Endovasc Surg 1986; 11: 324-329.

2. Costa M, Robbs JV. Abdominal aneurysm in a black population: A clinicopathological study. Br J Surg 1986; 73: 554-558

3. Decker CAG, Samson ID, Schmaman A. Abdominal aneurysm in South African Negroes due to intimomedial mucoid degeneration. Br J Surg 1977; 64: 513-516.

4. Steiner J, Thomas ID, et al. Aortopathies in Ugandan Africans. J Pathol 1973; 109: 295-305.

5. Sandhyamani S. Mucoid vasculopathy: vascular lesions in an autopsy study. Mod Pathol 1993; 6: 333-336.

6. Woolgar JD, Robbs JV, et al. Giant abdominal aortic aneurysm secondary to intimomedial mucoid degeneration. Postgrad Med J 2001; 77: 782

7. Cooper K. Extraaortic intimomedial mucoid degeneration: a clinicopathological study. Angiology 1993 44(6): $477-482$

8. Rutherford RB. Vascular Surgery. 2nd ed. Philadelphia: Saunders, 1984. 